\title{
Is Retroflexion Helpful in Detecting Adenomas in the Right Colon?: A Single Center Interim Analysis
}

\author{
Hyun Seok Lee, Seong Woo Jeon \\ Department of Internal Medicine, Kyungpook National University School of Medicine, Daegu, Korea
}

Background/Aims: Colonoscopy is less effective at screening for colorectal cancer in the right side of the colon. Retroflexion during colonoscopy is expected to improve the detection rate of colorectal adenomas. The aim of the present study was to evaluate the usefulness of retroflexion in the right-sided colon. Methods: From April to November 2013, a total of 398 patients were enrolled in this study. For each patient, a cap-assisted colonoscopic examination was performed. After cecal intubation, a forward view examination from the cecum up to the hepatic flexure was performed and all identified polyps were removed. The colonoscope was reinserted to the cecum, and a careful second forward view examination of the cecum to the hepatic flexure was performed, with removal of additionally identified polyps. The colonoscope was then reinserted to the cecum and retroflexed; a third colonoscopic examination was then performed to the hepatic flexure in retroflexion with removal of additional polyps. Total polyp numbers and characteristics were compared between the two forward view examinations and the retroflexion examination. Results: A successful retroflexion was performed in $90.2 \%$ of patients. A total of 213 polyps and 143 adenomas were detected in the right-sided colon using the routine method of examining the right colon twice in forward view. An additional 35 polyps and 24 adenomas were detected on retroflexion. Of these 35 polyps, 27 (77.1\%) were small-sized polyps $(\leq 5 \mathrm{~mm})$ and $24(71.4 \%)$ were adenomas. Finding additional adenomas using the retroflexion technique was associated with older age. Conclusions: Colonoscopic retroflexion is helpful in the detection of cecum and ascending colon adenomas, especially small-sized adenomas ( $\leq 5 \mathrm{~mm}$ ). It is particularly useful in older patients. (Intest Res 2015;13:326-331)

Key Words: Retroflexion; Colonoscopy; Adenoma

\section{INTRODUCTION}

Colonoscopy is one of the most effective methods in the screening and prevention of colorectal cancer, and it has been shown to lower the colorectal cancer death rate. ${ }^{1,2}$ To maximize colorectal cancer prevention using colonoscopy, it is crucial to increase the detection rate of colonic adenomas and the precursor lesions of colorectal cancer, and to reduce the miss rate. To achieve these goals, thorough ex-

Received October 13, 2014. Revised November 15, 2014.

Accepted December 30, 2014.

Correspondence to Seong Woo Jeon, Gastric Cancer Center, Kyungpook

National Univeristy Medical Center. 807 Hoguk-ro, Buk-gu, Daegu 41404,

Korea. Tel: +82-53-200-2602, Fax:+82-53-200-2027,E-mail: sw-jeon@

hanmail.net

Financial support: None. Conflict of interest: None. amination of the entire colonic mucosa is important. Prior to this procedure, a high cecal intubation rate, sufficient time for colonoscopy withdrawal, and proper cleansing of colon are required. ${ }^{3}$ Even when these basic requirements are fulfilled, the miss rate for small-sized colorectal adenomas and cancer is reported to reach up to $30 \%$ among experienced colonoscopists. ${ }^{4,5}$ One of the causes contributing to this miss rate is the fact that it is difficult to detect polyps hidden in the proximal side of the right-sided colon or flexures, especially in the medial aspect of the hepatic flexure. ${ }^{6-8}$ A retroflexion technique during colonoscopy is primarily used to increase the diagnostic yield of lesions in the distal rectum., ${ }^{9,10}$ Moreover, in some studies, retroflexion has been used in the right side of the colon to increase the diagnostic detection of polyps. However, these previous studies have reported that the implementation of the retroflexion technique in the right

\footnotetext{
๑ Copyright 2015. Korean Association for the Study of Intestinal Diseases. All rights reserved.

This is an Open Access article distributed under the terms of the Creative Commons Attribution Non-Commercial License (http://creativecommons.org/licenses/by-nc/4.0)

which permits unrestricted non-commercial use, distribution, and reproduction in any medium, provided the original work is properly cited.
} 
side of the colon did not result in a significant decrease in the miss rate of colonic adenomas. ${ }^{11,12}$ Therefore, this prospective study aimed to investigate the effectiveness and safety of retroflexion in improving the detection rate of polyps and adenomas in the right side of the colon.

\section{METHODS}

\section{Materials}

This study prospectively selected subjects who visited the Division of Gastroenterology, Kyungpook National University Medical Center for health screening or follow-up colonoscopy between April and November 2013. We defined no history of colon polyps as 'screening', and previous detection of colon polyps as 'surveillance.' We excluded patients younger than 18 years or older than 86 years, and those with a previous history of bowel resection, IBD or polyposis syndrome. This study was conducted after obtaining approval from the Kyungpook National University Medical Center internal review board (KNUMC_12-1036).

\section{Methods and Analysis}

The hood-cap (MH-466; Olympus Medical Systems, Tokyo, Japan) was attached to the tip of the colonoscope, and capassisted colonoscopy (CF-H260AL; Olympus Optical Co., Tokyo, Japan) was performed in all subjects. All procedures were conducted by 9 gastroenterologists: 3 experienced gastroenterologists, each who had over 5 years of experience and had performed more than 1,000 colonoscopies, and 6 inexperienced gastroenterologists.

Colon cleanliness was evaluated using an assessment of bowel preparation quality as suggested by The American Society for Gastrointestinal Endoscopy (ASGE). The scale was 1 (excellent) when mostly liquid stool was present and minimal suction was needed for a small amount of fluid; 2 (good), when mostly liquid stool was present and significant suction was needed for a small amount of fluid; 3 (fair), when a mixture of liquid and semisolid stool could be suctioned and/or washed out; and 4 (poor), when a mixture of semisolid and solid stool could not be suctioned or washed out. ${ }^{3,13}$ After insertion of the colonoscope up to the cecum, the colon including the cecum from the ascending colon (AC) to the hepatic flexure and medial folds were thoroughly inspected in the forward view. All polyps that were detected during the examination were removed and sent for histological examination. After removing any polyps found in the forward view in the right colon, a colonoscope was inserted into the cecum for a second time. Once again, the cecum from the AC to the hepatic flexure and medial folds were thoroughly examined in the forward view. Additional polyps were also removed and sent for histological examination. The colonoscope was then reinserted into the cecum for a third time and retroflexed from the cecum. With the use of a retroflexion technique in the right colon, we viewed the colon from the cecum to the hepatic flexure. Additional polyps were also removed and sent for histological examination. The hepatic flexure appeared blue because the liver was seen through the mucosa on retroflexion or the acute bends of the colonic lumen and dome-like shapes like the gastric fundus were observed in the proximal hepatic flexure. In all 3 examinations, the colonoscopy withdrawal time was sustained for at least 2 minutes to allow adequate diagnosis of polyps.

We intended to assess the additional yields of polyps and adenomas achieved on retroflexion by comparing the results of the 2 forward view examinations and the retroflexion examination. The polyp miss rate was computed as the number of polyps/adenomas detected on retroflexion among the total number of polyps/adenomas detected on the 3 examinations. The per-patient miss rate was computed as the number of patients with additional polyps detected on retroflexion among the total number of patients who underwent the examination. Failed retroflexion cases were included in the total number of patients for intention-to-treat analysis, and the causes of failure were recorded.

\section{Statistical Analysis}

Values were expressed as means and percentages, and continuous variables were presented as the mean \pm standard deviation. A paired $t$-test was conducted to analyze continuous variables and a chi-square test was performed to evaluate categorical variables. A logistic regression analysis was carried out to compare the relative risk of factors showing significance. Significant factors in univariate linear regression analysis were determined when these variables were found to be independent factors of prediction through multiple linear regression analysis. All statistical analyses were performed using SPSS version 14.0 (SPSS Inc., Chicago, IL, USA). The CI was set at $95 \%$, and $P$-values of less than 0.05 were considered statistically significant. 


\section{RESULTS}

\section{Retroflexion}

This study examined a total of 398 subjects (222 men and 176 women); the mean patient age was $56.0 \pm 12.7$ years (Table 1). There were no retroflexion-related complications in any subject. Right colon retroflexion was successfully conducted in 359 (90.2\%) cases; it was not performed in 39 $(9.8 \%)$ cases. The causes of the retroflexion failure in these 39 subjects were instrumental looping in 20 cases (5.0\%); angulation of the AC or narrowing colon in 13 cases (3.3\%); and complaints of pain in 6 cases (1.5\%). The bowel cleanliness of the AC was excellent in 325 subjects, good in 48, fair in 22 , and poor in 3 . There was no subject with inadequate bowel preparation quality.
Table 1. Baseline Characteristics of the Subjects

\begin{tabular}{lc}
\hline \multicolumn{1}{c}{ Characteristic } & $\begin{array}{c}\text { Enrolled subjects } \\
(\mathbf{n = 3 9 8 )}\end{array}$ \\
\hline Sex & \\
Male & $222(55.8)$ \\
Female & $176(44.2)$ \\
Age (yr) & $56.0 \pm 12.7$ \\
Height (cm) & $163.9 \pm 9.2$ \\
Weight (kg) & $65.0 \pm 11.4$ \\
Reason for colonoscopy & \\
Screening (no history of colon polyps) & $308(77.4)$ \\
Surveillance (past history of colon polyps) & $90(22.6)$ \\
\hline
\end{tabular}

Values are presented as mean \pm SD or $n(\%)$.

Table 2. Comparison Between Forward View Examination and Retroflexion Examination

\begin{tabular}{|c|c|c|c|c|c|c|}
\hline Variable & Total $^{*}$ & $\begin{array}{c}\text { First } \\
\text { forward view }\end{array}$ & $\begin{array}{c}\text { Second } \\
\text { forward view }\end{array}$ & $\begin{array}{c}\text { Subtotal } \\
\text { forward view }^{+}\end{array}$ & $\begin{array}{c}\text { Retroflexion } \\
\text { (third examination) }\end{array}$ & $\begin{array}{c}P \text {-value } \\
\text { (subtotal vs. total) }\end{array}$ \\
\hline \multicolumn{7}{|l|}{ Polyp } \\
\hline \multicolumn{7}{|l|}{ Size } \\
\hline$\leq 5 \mathrm{~mm}$ & 167 (67.3) & 117 (63.9) & 23 (76.7) & $140(65.7)$ & 27 (77.1) & $<0.001$ \\
\hline$\geq 10 \mathrm{~mm}$ & 40 (16.1) & $32(17.5)$ & $3(10.0)$ & 35 (16.4) & $5(14.3)$ & 0.025 \\
\hline \multicolumn{7}{|l|}{ Location } \\
\hline Cecum and proximal $A C$ & $98(39.5)$ & $79(43.2)$ & $14(46.7)$ & $93(43.7)$ & $5(14.3)$ & 0.025 \\
\hline Mid AC & $71(28.6)$ & $53(29.0)$ & 7 (23.3) & $60(28.2)$ & $11(31.4)$ & 0.001 \\
\hline Non-polypoid & $92(37.1)$ & $69(37.7)$ & $13(43.3)$ & $82(38.5)$ & $10(28.6)$ & 0.001 \\
\hline \multicolumn{7}{|l|}{ Adenoma } \\
\hline $\begin{array}{l}\text { Number } \\
\text { (adenoma to polyp ratio) }\end{array}$ & 167 (67.3) & 117 (63.9) & 26 (86.7) & 143 (67.1) & 24 (68.6) & $<0.001$ \\
\hline \multicolumn{7}{|l|}{ Size } \\
\hline$\leq 5 \mathrm{~mm}$ & 109 (65.3) & $71(60.7)$ & 20 (76.9) & 91 (63.6) & $18(75.0)$ & $<0.001$ \\
\hline $6-9 \mathrm{~mm}$ & $29(17.4)$ & $23(19.7)$ & $3(11.5)$ & $26(18.2)$ & $3(12.5)$ & 0.083 \\
\hline$\geq 10 \mathrm{~mm}$ & $29(17.4)$ & 23 (19.7) & $3(11.5)$ & 26 (18.2) & $3(12.5)$ & 0.083 \\
\hline
\end{tabular}

Values are presented as $n(\%)$.

*First forward view, second forward view, and retroflexion.

${ }^{\dagger}$ First forward view and second forward view, not retroflexion.

$A C$, ascending colon. 
Table 3. Multiple Logistic Regression Analysis of Predictors for the Detection of Polyps/Adenomas Using Right-Sided Colon Retroflexion

\begin{tabular}{|c|c|c|c|c|c|c|}
\hline \multirow{2}{*}{ Variable } & \multicolumn{3}{|c|}{ Presence of polyps in retroflexion } & \multicolumn{3}{|c|}{ Presence of adenomas in retroflexion } \\
\hline & OR & $95 \% \mathrm{Cl}$ & $P$-value & OR & $95 \% \mathrm{Cl}$ & $P$-value \\
\hline Presence of polyp on forward view & 0.556 & $0.217-1.424$ & 0.221 & 0.560 & $0.186-1.683$ & 0.302 \\
\hline Male & 1.632 & $0.660-4.035$ & 0.289 & 1.957 & $0.646-5.935$ & 0.235 \\
\hline Female & Reference & & & Reference & & \\
\hline \multicolumn{7}{|l|}{ Indication } \\
\hline Screening* & 1.022 & $0.357-2.921$ & 0.968 & 0.846 & $0.254-2.814$ & 0.785 \\
\hline Surveillance $^{+}$ & Reference & & & Reference & & \\
\hline
\end{tabular}

*No history colon polyps.

${ }^{\dagger}$ Past history of colon polyps.

\section{Polyps}

Of the 213 polyps detected in the AC on the 2 forward view examinations before retroflexion, the polyp size was less than $5 \mathrm{~mm}$ for $140(65.7 \%)$ polyps, $6-9 \mathrm{~mm}$ for 38 (17.8\%) polyps, and greater than $10 \mathrm{~mm}$ for 35 (16.4\%) polyps. Of the 35 additional polyps found on retroflexion, 27 polyps (77.1\%) were less than $5 \mathrm{~mm}$ in size, 3 (8.6\%) were 6-9 $\mathrm{mm}$ in size, and 5 (14.3\%) were greater than $10 \mathrm{~mm}$ in size (Table 2). During the two forward view examinations, 93 (43.7\%) polyps were detected in the proximal AC including the cecum, 60 (28.2\%) polyps in the mid-AC, and $60(28.2 \%)$ polyps in the distal AC. The detection rate in the distal AC was higher using retroflexion (19 polyps, 54.3\%). Polyp shape, according to the forward view examinations, was polypoid for 131 (61.5\%) polyps, and non-polypoid for 82 (38.5\%) polyps. Polyp shape, as determined on retroflexion, was polypoid for 25 (71.4\%) polyps, and non-polypoid for 10 (28.6\%) polyps. Regardless of the polyp shape, the additional number of detected polyps on retroflexion was statistically significantly higher than that detected on the forward view examinations $(P<0.001$ for polypoid polyps, $P=0.001$ for non-polypoid polyp).

\section{Adenomas}

Of all polyps detected in the AC on the 2 forward view examinations prior to retroflexion, $143(67.1 \%)$ were adenomas. Of these, 91 (63.6\%) were less than $5 \mathrm{~mm}$ in size, $26(18.2 \%)$ were $6-9 \mathrm{~mm}$ in size, and $26(18.2 \%)$ were larger than $10 \mathrm{~mm}$ in size. Of the additional polyps identified on retroflexion, 24 (68.6\%) were adenomas. Of these adenomas, $18(75.0 \%)$ were less than $5 \mathrm{~mm}$ in size, $3(12.5 \%)$ were $6-9$ $\mathrm{mm}$ in size, and $3(12.5 \%)$ were larger than $10 \mathrm{~mm}$ in size. The number of detected adenomas less than $5 \mathrm{~mm}$ in size was statistically significantly higher with retroflexion than in the forward view examinations $(P<0.001)$.

We performed univariate and multiple logistic regression analyses of polyp and adenoma predictors of detection in right-sided colon retroflexion. Of all the possible predictors, old age was found to be statistically significant (Polyps: OR, 1.057; 95\% CI, 1.017-1.098; $P=0.005$; Adenomas: OR, 0.081; 95\% CI, $1.029-1.135 ; P=0.002$ ) (Table 3 ).

\section{DISCUSSION}

Adenomas are considered precursor lesions of colorectal cancer, and the risk of colorectal cancer can be reduced by removing adenomas. ${ }^{14}$ Recent studies have suggested that colonoscopies are less effective in preventing colorectal cancer in the proximal colon compared with the distal colon. ${ }^{15-17}$ This finding is attributable to the relative higher number of small, flat polyps found mainly in the right side of the colon. ${ }^{18,19}$ Some studies have revealed that cap-assisted colonoscopy is helpful in increasing the yield of detected colon polyps and adenomas. ${ }^{12,20,21}$ In addition, previous studies have found that the use of chromoendoscopy ${ }^{22,23}$ and narrow band imaging ${ }^{24,25}$ during colonoscopy can detect a significantly large number of polyps or adenomas. Hence, this study evaluated the additional detected yields of polyps and adenomas achieved using colonoscopic retroflexion in order to improve the detection rate of polyps and adenomas in the right colon. To reduce differences in the detection rate caused by the use of hood caps, cap-assisted colonoscopy was performed in all subjects. In this prospective study on 
398 subjects, right colon retroflexion was successfully conducted in $90.2 \%$ of the subjects without any complications. A total of 213 polyps were detected in 114 (28.6\%) subjects by examining the colon twice in forward view. Subsequently, a total of 35 polyps were additionally found in 34 (8.5\%) subjects after retroflexion. Additional polyps that were undetected on the two forward view examinations were found in 13 (4.6\%) out of 284 subjects during retroflexion.

Harrison et al. (2004) conducted colonoscopies in 100 patients to examine the effect of retroflexion on the adenoma miss rate in the right colon. ${ }^{11}$ After forward-view examinations in the right colon, either a second forward view or a retroflexion examination was randomly performed after the initial colonoscopy, and the difference in the adenoma miss rate between forward view and retroflexion examinations was analyzed. The polyp miss rate was $36.8 \%$ and $38.1 \%$ in forward view and retroflexion examinations, respectively, and the adenoma miss rate was $33.3 \%$ and $23.7 \%$, respectively, showing no significant difference. The second forward view and retroflexion examinations showed no difference in the adenoma miss rate, and these findings implied that there was no benefit to an additional retroflexion examination. ${ }^{11}$ To determine the potential usefulness of an additional retroflexion examination in the present study, the right colon was examined in forward view at the second examination, and then with retroflexion at the third examination. Based on the identified polyp yields using retroflexion, the polyp miss rate was $14.1 \%$ in the 2 forward view examinations. In contrast to the results of previous studies, this study revealed that retroflexion was effective in identifying considerably more right colon polyps than the 2 forward view examinations.

In a study on polyp miss rate using right-sided colon retroflexion by Hewett and Rex (2011), investigators excised polyps on the right side of the colon using forward view examinations, and then analyzed polyps that were additionally detected using retroflexion beginning from the cecum. ${ }^{26}$ Retroflexion was successfully performed in $94.4 \%$ of cases, and polyp and adenoma miss rates were $9.7 \%$ and $9.8 \%$, respectively. The predictors of finding additional polyps or adenomas in the right colon using retroflexion are reported to be old age, male sex, and polyp detection on forward view examination. Through additional retroflexion, polyps were found in $2.2 \%$ of all patients in whom no polyps had been detected in the first forward view examination. However, this previous research was not a comparative study of forward view and retroflexion techniques; it was about success rate, safety and the polyp detection rate of right colon retroflexion, irrespective of its effectiveness. ${ }^{26}$ In the present study, the polyp miss rate was $14.1 \%$ and the adenoma miss rate was $14.3 \%$. With additional retroflexion, polyps were found in $4.6 \%$ of all cases with no polyp detection in the first forward view examination. This outcome implies that retroflexion is able to detect right colon polyps that are rarely observed with forward view colonoscopy. As polyps detected during retroflexion are rarely seen in repeated forward view examinations, the additional retroflexion procedure is thought to be beneficial in improving the detection of polyps located at the colonic mucosa blind spot (mucosal folds in the proximal aspect and the medial aspect of the hepatic flexure). To minimize polyp yields during additional retroflexion, each forward view examination was carefully performed for at least 2 minutes to allow sufficient observation time.

Using the retroflexion technique, additionally detected polyps and adenomas less than $5 \mathrm{~mm}$ in size accounted for $77.1 \%$ and $75.0 \%$ of observations, respectively. Large-sized polyps or adenomas were mostly identified during forward view examinations, and small-sized polyps undetected in the first forward view examination were observed at a considerably higher rate during subsequent retroflexion. A significant predicting factor for the detection of additional polyps or adenomas using retroflexion was found to be old age.

The formation of a loop during retroflexion in the right colon makes colonoscopy insertion difficult. More loops were formed during the 2 forward view examinations than the retroflexion examinations; these loops were obstacles to complete examination.

One of the limitations of this study was the relatively small sample size, but further research is underway to include more subjects. Another limitation is that this study involved a number of inexperienced colonoscopists who did not adequately perform the retroflexion technique. Unlike previous studies, this study was unable to compare the results by randomly allocating forward view or retroflexion as a follow-up examination. Moreover, it may be difficult to completely exclude operator intention on additional polyp detection using retroflexion. To overcome this, we investigated whether it is possible to detect additional polyps that were undetected on repeated forward view examinations. Additionally detected polyps and adenomas less than $5 \mathrm{~mm}$ in size accounted for a considerably larger percentage of the retroflexion-detected events, and further studies are warranted to examine the clinical significance of the additional detection of these small adenomas.

In conjunction with our previous findings, these results suggest that there may be spots in the right colon where polyps are rarely detected despite repeated forward view 
examinations. The retroflexion maneuver is effective in increasing the detected number of polyps missed on forward view examinations and particularly helpful for individuals of older age.

\section{REFERENCES}

1. Rex DK, Johnson DA, Lieberman DA, Burt RW, Sonnenberg A. Colorectal cancer prevention 2000: screening recommendations of the American College of Gastroenterology. American College of Gastroenterology. Am J Gastroenterol 2000;95:868877.

2. Manser CN, Bachmann LM, Brunner J, Hunold F, Bauerfeind P, Marbet UA. Colonoscopy screening markedly reduces the occurrence of colon carcinomas and carcinoma-related death: a closed cohort study. Gastrointest Endosc 2012;76:110-117.

3. Chun CG, Kim HG, Jeon SR, Ko BM, Lee BH, Kim JO. Analysis of colonoscopy quality in clinical practices of Korea: cohort study of patients referred to tertiary hospital after colonoscopy examinations. Intest Res 2013;11:198-203.

4. Deenadayalu VP, Chadalawada V, Rex DK. 170 degrees wideangle colonoscope: effect on efficiency and miss rates. Am J Gastroenterol 2004;99:2138-2142.

5. van Rijn JC, Reitsma JB, Stoker J, Bossuyt PM, van Deventer SJ, Dekker E. Polyp miss rate determined by tandem colonoscopy: a systematic review. Am J Gastroenterol 2006;101:343-350.

6. Rex DK. Maximizing detection of adenomas and cancers during colonoscopy. Am J Gastroenterol 2006;101:2866-2877.

7. Barclay RL, Vicari JJ, Doughty AS, Johanson JF, Greenlaw RL. Colonoscopic withdrawal times and adenoma detection during screening colonoscopy. N Engl J Med 2006;355:2533-2541.

8. Rex DK. Colonoscopic withdrawal technique is associated with adenoma miss rates. Gastrointest Endosc 2000;51:33-36.

9. Hanson JM, Atkin WS, Cunliffe WJ, et al. Rectal retroflexion: an essential part of lower gastrointestinal endoscopic examination. Dis Colon Rectum 2001;44:1706-1708.

10. Cutler AF, Pop A. Fifteen years later: colonoscopic retroflexion revisited. Am J Gastroenterol 1999;94:1537-1538.

11. Harrison M, Singh N, Rex DK. Impact of proximal colon retroflexion on adenoma miss rates. Am J Gastroenterol 2004;99:519-522.

12. Kwon SC, Choi SW, Choi SH, et al. The effect of indigocarmine on improvement of the polyp detection rate during colonoscopic examination with hood cap. Intest Res 2014;12:60-65.

13. Rex DK, Petrini JL, Baron TH, et al. Quality indicators for colonoscopy. Am J Gastroenterol 2006;101:873-885.
14. Winawer SJ, Zauber AG, Ho MN, et al. Prevention of colorectal cancer by colonoscopic polypectomy. The National Polyp Study Workgroup. N Engl J Med 1993;329:1977-1981.

15. Lakoff J, Paszat LF, Saskin R, Rabeneck L. Risk of developing proximal versus distal colorectal cancer after a negative colonoscopy: a population-based study. Clin Gastroenterol Hepatol 2008;6:1117-1121.

16. Singh H, Nugent Z, Demers AA, Kliewer EV, Mahmud SM, Bernstein CN. The reduction in colorectal cancer mortality after colonoscopy varies by site of the cancer. Gastroenterology 2010;139:1128-1137.

17. Singh H, Nugent Z, Mahmud SM, Demers AA, Bernstein CN. Predictors of colorectal cancer after negative colonoscopy: a population-based study. Am J Gastroenterol 2010;105:663-673.

18. Hetzel JT, Huang CS, Coukos JA, et al. Variation in the detection of serrated polyps in an average risk colorectal cancer screening cohort. Am J Gastroenterol 2010;105:2656-2664.

19. Soetikno RM, Kaltenbach T, Rouse RV, et al. Prevalence of nonpolypoid (flat and depressed) colorectal neoplasms in asymptomatic and symptomatic adults. JAMA 2008;299:1027-1035.

20. Westwood DA, Alexakis N, Connor SJ. Transparent cap-assisted colonoscopy versus standard adult colonoscopy: a systematic review and meta-analysis. Dis Colon Rectum 2012;55:218-225.

21. Ng SC, Tsoi KK, Hirai HW, et al. The efficacy of cap-assisted colonoscopy in polyp detection and cecal intubation: a metaanalysis of randomized controlled trials. Am J Gastroenterol 2012;107:1165-1173.

22. Hurlstone DP, Cross SS, Slater R, Sanders DS, Brown S. Detecting diminutive colorectal lesions at colonoscopy: a randomised controlled trial of pan-colonic versus targeted chromoscopy. Gut 2004;53:376-380.

23. Pohl J, Schneider A, Vogell H, Mayer G, Kaiser G, Ell C. Pancolonic chromoendoscopy with indigo carmine versus standard colonoscopy for detection of neoplastic lesions: a randomised two-centre trial. Gut 2011;60:485-490.

24. Hirata M, Tanaka S, Oka S, et al. Magnifying endoscopy with narrow band imaging for diagnosis of colorectal tumors. Gastrointest Endosc 2007;65:988-995.

25. Inoue T, Murano M, Murano N, et al. Comparative study of conventional colonoscopy and pan-colonic narrow-band imaging system in the detection of neoplastic colonic polyps: a randomized, controlled trial. J Gastroenterol 2008;43:45-50.

26. Hewett DG, Rex DK. Miss rate of right-sided colon examination during colonoscopy defined by retroflexion: an observational study. Gastrointest Endosc 2011;74:246-252. 